PROCEEDINGS OF THE

AMERICAN MATHEMATICAL SOCIETY

Volume 127, Number 12, Pages 3557-3564

S 0002-9939(99)04949-7

Article electronically published on May 13, 1999

\title{
NONLINEAR ALTERNATIVES FOR MULTIVALUED MAPS WITH APPLICATIONS TO OPERATOR INCLUSIONS IN ABSTRACT SPACES
}

\author{
DONAL O'REGAN
}

(Communicated by Dale Alspach)

\begin{abstract}
A nonlinear alternative of Leray-Schauder type is presented for condensing operators with closed graph. We will then use this theorem to establish new existence principles for differential and integral inclusions in Banach spaces.
\end{abstract}

\section{INTRODUCTION}

In this paper we establish new nonlinear alternatives of Leray-Schauder type for multivalued maps. Our theory was motivated by the following alternative.

Theorem 1.1. Let $E$ be a Banach space and let $U$ be an open subset of $E$ with $0 \in U$. Suppose

$$
\left\{\begin{array}{l}
F: \bar{U} \rightarrow C(E) \text { has closed graph; here } C(E) \text { denotes } \\
\text { the family of nonempty, closed, convex subsets of } E
\end{array}\right.
$$

and

$$
F: \bar{U} \rightarrow C(E) \text { is compact }
$$

hold. Then either

(A1). F has a fixed point in $\bar{U}$; or

(A2). there exists $u \in \partial U$ and $\lambda \in(0,1)$ with $u \in \lambda F(u)$.

The proof follows immediately from the nonlinear alternative for upper semicontinuous (u.s.c.) maps [5] once we notice that (1.1), (1.2) and [1, page 465] imply $F: \bar{U} \rightarrow C(E)$ is u.s.c. However when we examine differential and integral inclusions in abstract spaces (when the dimension is infinite) it is of interest to replace (1.2) with the less restrictive condition

$$
\left\{\begin{array}{l}
F: \bar{U} \rightarrow C(E) \text { is a condensing map with } \\
F(\bar{U}) \text { a subset of a bounded set in } E .
\end{array}\right.
$$

In this case it is not clear how to apply the nonlinear alternative for u.s.c. maps since $F: \bar{U} \rightarrow C(E)$ may not necessarily be u.s.c. (note $F(\bar{U})$ is not necessarily relatively compact so [1, page 465] does not apply). In the literature $[3,8,9]$ the authors were able to establish fixed points to multivalued operators of the above

Received by the editors September 10, 1997 and, in revised form, February 13, 1998.

1991 Mathematics Subject Classification. Primary 47H10, 54C60, 54H25.

(C)1999 American Mathematical Society 
type by using other approaches; in particular using Ky Fan's fixed point theorem together with some tricks. However an applicable existence principle of Theorem 1.1 type (when $\operatorname{dim} E=\infty$ ) has not appeared with (1.3) replacing (1.2). We fill this gap in the literature in this paper. To do so we first establish some new nonlinear alternatives of Leray-Schauder type (see Theorem 2.1 and Theorem 2.2); standard alternatives in the literature (see Corollary 2.1 and Corollary 2.2) can be deduced from our results.

To conclude the introduction we gather together some definitions. In this paper $2^{E}$ denotes the family of nonempty subsets of $E, C D(E)$ the family of nonempty, closed, acyclic (see [4] ) subsets of $E$ and $C K(E)$ the family of nonempty, compact, acyclic subsets of $E$. Let $X$ be a Banach space and $\Omega_{X}$ the bounded subsets of $X$. The Kuratowskii measure of noncompactness is the map $\alpha: \Omega_{X} \rightarrow[0, \infty]$ defined by

$$
\alpha(Z)=\inf \left\{\epsilon>0: Z \subseteq \bigcup_{i=1}^{n} Z_{i} \text { and } \operatorname{diam}\left(Z_{i}\right) \leq \epsilon\right\} ; \text { here } Z \in \Omega_{X} .
$$

Let $X_{1}$ and $X_{2}$ be Banach spaces. A multivalued map $F: Y \subseteq X_{1} \rightarrow X_{2}$ is said to be $\alpha$-Lipschitzian if it maps bounded sets into bounded sets and if there exists a constant $k \geq 0$ with $\alpha(F(Z)) \leq k \alpha(Z)$ for all bounded sets $Z \subseteq Y$. We call $F$ a condensing map if $F$ is $\alpha$-Lipschitzian with $k=1$ and $\alpha(F(Z))<\alpha(Z)$ for all bounded sets $Z \subseteq Y$ with $\alpha(Z) \neq 0$.

\section{FiXed POINT THEORY}

For convenience we assume throughout this section that $E$ is a Banach space (the extension to the case when $E$ is a Fréchet space is clear). From an application point of view we are interested in multivalued maps with closed graph. First however we consider maps which are also u.s.c. Our first result was motivated by ideas in $[2,6,7]$.

Theorem 2.1. Let $E$ be a Banach space (or more generally a Fréchet space) with $U$ an open subset of $E$ and $x_{0} \in U$.

(a). Suppose the following conditions are satisfied:

$$
F: \bar{U} \rightarrow C K(E) \text { is u.s.c. }
$$

and

$$
\left\{\begin{array}{l}
\text { there exists } X \subseteq E \text { with } X=\overline{c o}\left(\left\{x_{0}\right\} \cup F(X \cap \bar{U})\right) \\
\text { and } X \text { is compact. }
\end{array}\right.
$$

Then either

(A1). $F$ has a fixed point in $\bar{U}$; or

(A2). there exist $u \in \partial U$ and $\lambda \in(0,1)$ with $u \in \lambda F(u)+(1-\lambda)\left\{x_{0}\right\}$.

(b). Suppose the following conditions are satisfied:

$$
F: \bar{U} \rightarrow E \text { is single valued and continuous }
$$

and

$$
\left\{\begin{array}{l}
\text { there exists } W \subseteq E \text { with } W=c o\left(\left\{x_{0}\right\} \cup F(W \cap U)\right) \\
\text { and } \bar{W} \text { is compact. }
\end{array}\right.
$$


Then either

(A1). F has a fixed point in $\bar{U}$; or

(A2). there exist $u \in \partial U$ and $\lambda \in(0,1)$ with $u=\lambda F(u)+(1-\lambda) x_{0}$.

Proof. Without loss of generality assume $x_{0}=0$.

(a). Suppose $(A 2)$ does not occur. Also without loss of generality assume $F$ has no fixed points in $\partial U$ (otherwise we are finished, i.e. ( $A 1)$ occurs). Let

$$
H=\{x \in \bar{U}: x \in \lambda F(x) \text { for some } \lambda \in[0,1]\} .
$$

Now $H \neq \emptyset$ since $0 \in H$. Also $H$ is closed. To see this let $\left(x_{n}\right)$ be a sequence in $H$ (i.e. $x_{n} \in \lambda_{n} F\left(x_{n}\right)$ for some $\left.\lambda_{n} \in[0,1]\right)$ with $x_{n} \rightarrow x_{0} \in \bar{U}$. Without loss of generality assume $\lambda_{n} \rightarrow \lambda_{0} \in[0,1]$. Let $R: \bar{U} \times[0,1] \rightarrow C K(E)$ be defined by $R(x, \lambda)=\lambda F(x)$. Now it's easy to see [7] that $R: \bar{U} \times[0,1] \rightarrow C K(E)$ is u.s.c. Also since $\left(x_{n}, \lambda_{n}\right)$ is a sequence in $\bar{U} \times[0,1]$ with $\left(x_{n}, \lambda_{n}\right) \rightarrow\left(x_{0}, \lambda_{0}\right)$ and $x_{n} \in R\left(x_{n}, \lambda_{n}\right)$, we see from [10] that $x_{0} \in R\left(x_{0}, \lambda_{0}\right)$, i.e. $x_{0} \in H$. Thus $H$ is closed.

Remark 2.1. It is of interest to note (see Theorem 2.2) that if $F: \bar{U} \rightarrow C K(E)$ is u.s.c. is replaced by $F: \bar{U} \rightarrow C D(E)$ (or $2^{E}$ ) has closed graph, then once again $H$ is closed. To see this let $x_{n}$ and $\lambda_{n}$ be as above with $x_{n} \rightarrow x_{0} \in \bar{U}$ and $\lambda_{n} \rightarrow \lambda_{0} \in[0,1]$. Without loss of generality assume $\lambda_{0} \in(0,1]$. Since $x_{n} \in H$, there exists $y_{n} \in F\left(x_{n}\right)$ with $x_{n}=\lambda_{n} y_{n}$. Now $x_{n} \rightarrow x_{0}$ and $y_{n} \rightarrow \frac{1}{\lambda_{0}} x_{0}$. The closedness of $F$ implies $\frac{1}{\lambda_{0}} x_{0} \in F\left(x_{0}\right)$ so $x_{0} \in H$ [alternatively it is easy to see that $R: \bar{U} \times[0,1] \rightarrow C D(E)$ (or $2^{E}$ ), given above, has closed graph so it is immediate that $H$ is closed].

Now there exists (see (2.2)) $X \subseteq E$ with $X=\overline{c o}(\{0\} \cup F(X \cap \bar{U}))$ and $X$ is compact. Notice since $H$ is closed in $E$, then $H \cap X$ is closed in $X$.

Remark 2.2. In fact since $X$ is compact, we have that $H \cap X$ is compact.

Thus $H \cap X$ and $\partial_{X}(U \cap X)$ (the boundary of $U \cap X$ in $\left.X\right)$ are closed in $X$. We now claim $H \cap X$ and $\partial_{X}(U \cap X)$ are disjoint. To see this notice

$$
\partial_{X}(U \cap X)=\overline{U \cap X} \backslash(U \cap X) \subseteq \bar{U} \cap X \backslash(U \cap X)=(\bar{U} \backslash U) \cap X=\partial U \cap X
$$

and so

$$
(H \cap X) \cap \partial_{X}(U \cap X) \subseteq(H \cap X) \cap(\partial U \cap X) .
$$

Now $H \cap \partial U=\emptyset$ together with (2.5) implies $H \cap X$ and $\partial_{X}(U \cap X)$ are disjoint. There exists a continuous function $\mu: X \rightarrow[0,1]$ with

$$
\mu(H \cap X)=1 \text { and } \mu\left(\partial_{X}(U \cap X)\right)=0 .
$$

Define the map $J$ by

$$
J(x)=\left\{\begin{array}{l}
\mu(x) F(x), \quad x \in \overline{U \cap X}, \\
\{0\}, \quad x \in X \backslash \overline{U \cap X} .
\end{array}\right.
$$

Remark 2.3. Note $\overline{U \cap X}=(U \cap X) \cup \partial_{X}(U \cap X)$ and $\mu(x)=0$ if $x \in \partial_{X}(U \cap X)$.

It is easy to see that $J: X \rightarrow C K(E)$ is u.s.c. In fact $J: X \rightarrow C K(X)$. To see this notice

$J(X) \subseteq c o(\{0\} \cup F(\overline{U \cap X})) \subseteq c o(\{0\} \cup F(\bar{U} \cap X)) \subseteq \overline{c o}(\{0\} \cup F(\bar{U} \cap X))=X$. 
Thus $J: X \rightarrow C K(X)$ is u.s.c. and $X$ is compact. Now [4] implies that there exists $x \in X$ with $x \in J(x)$. Now since $0 \in U$ we have $x \in \overline{U \cap X}$ so

$$
x \in \mu(x) F(x) \text { with } \quad x \in \overline{U \cap X} \text {. }
$$

Thus $x \in \lambda F(x)$ with $0 \leq \lambda=\mu(x) \leq 1$. As a result $x \in H$ and so $x \in H \cap X$. Consequently $\mu(x)=1$ and so $x \in F(x)$. The proof is complete.

(b). Suppose $(A 2)$ does not occur and $F$ has no fixed points in $\partial U$. Let

$$
H=\{x \in \bar{U}: x=\lambda F(x) \text { for some } \lambda \in[0,1]\} .
$$

As above $H \neq \emptyset$ and $H$ is closed. Also there exists (see (2.4)) $W \subseteq E$ with

$$
W=c o(\{0\} \cup F(W \cap U))
$$

and $\bar{W}$ is compact. As in (a), $H \cap \bar{W}$ and $\partial_{\bar{W}}(U \cap \bar{W})$ are closed in $\bar{W}$ with $H \cap \bar{W}$ and $\partial_{\bar{W}}(U \cap \bar{W})$ disjoint. Thus there exists a continuous function $\mu: \bar{W} \rightarrow[0,1]$ with

$$
\mu(H \cap \bar{W})=1 \text { and } \mu\left(\partial_{\bar{W}}(U \cap \bar{W})\right)=0 .
$$

Define the map $J$ by

$$
J(x)=\left\{\begin{array}{l}
\mu(x) F(x), \quad x \in \overline{U \cap \bar{W}}, \\
0, \quad x \in \bar{W} \backslash \overline{U \cap \bar{W}} .
\end{array}\right.
$$

Notice $J: \bar{W} \rightarrow E$ is continuous. Also $J(\bar{W}) \subseteq \bar{W}$. To see this notice

$$
J(W) \subseteq \operatorname{co}(\{0\} \cup F(U \cap W))=W
$$

since if $x \in W$ and $x \in \partial_{\bar{W}}(U \cap \bar{W})$ or $x \in \bar{W} \backslash(\overline{U \cap \bar{W}})$, then $J(x)=0$ (the only other case is if $x \in W$ and $x \in U \cap \bar{W}$ i.e. $x \in U \cap W)$. Now $J(W) \subseteq W$ together with the fact that $J$ is continuous yields

$$
J(\bar{W}) \subseteq \overline{J(W)} \subseteq \bar{W} .
$$

Thus $J: \bar{W} \rightarrow \bar{W}$ is continuous and $\bar{W}$ is compact. Now Schauder's fixed point theorem implies that there exists $x \in X$ with $x=J(x)$. As in (a) we can easily deduce that $x=F(x)$.

Remark 2.4. Notice from the proof in (b), it is possible to replace the condition that $F$ is single valued and continuous (see (2.3)) with any multivalued u.s.c. map $F: \bar{U} \rightarrow C K(E)$ provided conditions are put on $F$ to guarantee that $J(\bar{W}) \subseteq$ $\overline{J(W)}$.

Before we prove the fixed point theorem that we will use in our applications, let us deduce from Theorem 2.1 some well known results.

Corollary 2.1 (Mönch). Let $E$ be a Banach space with $U$ an open subset of $E$ and $x_{0} \in U$. Suppose $F: \bar{U} \rightarrow E$ is continuous and that

if $C \subseteq \bar{U}$ is countable and $C \subseteq \overline{c o}\left(\left\{x_{0}\right\} \cup F(C)\right)$, then $\bar{C}$ is compact

holds. Then either

(A1). F has a fixed point in $\bar{U}$; or

(A2). there exist $u \in \partial U$ and $\lambda \in(0,1)$ with $u=\lambda F(u)+(1-\lambda) x_{0}$.

Proof. The result follows immediately from Theorem 2.1 (b) since a standard argument (see [2, page 204] or [6, page 992]) establishes (2.4). 
Corollary 2.2. Let $E$ be a Banach space with $U$ an open, convex subset of $E$ and $x_{0} \in U$. Suppose $F: \bar{U} \rightarrow C K(E)$ is a u.s.c., condensing map with $F(\bar{U})$ a subset of a bounded set in $E$. Then either

(A1). F has a fixed point in $\bar{U}$; or

(A2). there exist $u \in \partial U$ and $\lambda \in(0,1)$ with $u \in \lambda F(u)+(1-\lambda)\left\{x_{0}\right\}$.

Proof. The result follows immediately from Theorem 2.1 (a). We need only check (2.2). Notice [4, page 19] implies that there exists a closed, convex set $X$ with $x_{0} \in X$ and

$$
X=\overline{c o}\left(F(\bar{U} \cap X) \cup\left\{x_{0}\right\}\right) .
$$

We claim $\bar{X}=X$ is compact. To see this first notice $\alpha(\bar{U} \cap X)=0$ since if not, then

$$
\alpha(X)=\alpha\left(\overline{c o}\left(F(\bar{U} \cap X) \cup\left\{x_{0}\right\}\right)\right)=\alpha(F(\bar{U} \cap X))<\alpha(\bar{U} \cap X) \leq \alpha(X),
$$

a contradiction. Thus $\alpha(\bar{U} \cap X)=0$.

Remark 2.5. Notice $F(\bar{U} \cap X) \subseteq F(\bar{U})$ is bounded and as a result $X$ (and also $\bar{U} \cap X)$ is bounded.

Also notice

$$
\alpha(X)=\alpha\left(\overline{c o}\left(F(\bar{U} \cap X) \cup\left\{x_{0}\right\}\right)\right)=\alpha(F(\bar{U} \cap X)) \leq \alpha(\bar{U} \cap X)=0 .
$$

Thus $\bar{X}=X$ is compact so $(2.2)$ is true.

Remark 2.6. There is an obvious analogue of Corollary 2.2 if $E$ is a Fréchet space; we leave the proof to the reader.

Our next result was motivated by applications that occur in differential and integral inclusions (see Section 3).

Theorem 2.2. Let $E$ be a Banach space with $U$ an open, convex subset of $E$ and $x_{0} \in U$. Suppose

$$
F: \bar{U} \rightarrow C D(E) \text { has closed graph }
$$

and

$$
\left\{\begin{array}{l}
F: \bar{U} \rightarrow C D(E) \text { is a condensing map with } \\
F(\bar{U}) \text { a subset of a bounded set in } E
\end{array}\right.
$$

hold. Then either

(A1). F has a fixed point in $\bar{U}$; or

(A2). there exist $u \in \partial U$ and $\lambda \in(0,1)$ with $u \in \lambda F(u)+(1-\lambda)\left\{x_{0}\right\}$.

Remark 2.7. There is an obvious analogue of Theorem 2.2 when $E$ is a Fréchet space.

Proof. Without loss of generality assume $x_{0}=0$. Suppose $(A 2)$ does not occur and $F$ has no fixed points in $\partial U$. Let

$$
H=\{x \in \bar{U}: x \in \lambda F(x) \text { for some } \lambda \in[0,1]\} .
$$

As in Theorem 2.1 (see Remark 2.1), $H \neq \emptyset$ is closed. Also as in Corollary 2.2 there exists $X \subseteq E$ with $X=\overline{c o}(F(\bar{U} \cap X) \cup\{0\})$ and $X$ is compact. As in Theorem 2.1 (a), $H \cap X$ and $\partial_{X}(U \cap X)$ are closed in $X$ with $H \cap X$ and $\partial_{X}(U \cap X)$ disjoint. There exists a continuous function $\mu: X \rightarrow[0,1]$ with

$$
\mu(H \cap X)=1 \text { and } \mu\left(\partial_{X}(U \cap X)\right)=0 .
$$


Let's look at $\left.F\right|_{\overline{U \cap X}}$, i.e. $F: \overline{U \cap X} \rightarrow C D(E)$. Now (2.7) implies $\left.F\right|_{\overline{U \cap X}}$ has closed graph. This together with the fact that $\overline{U \cap X}$ is compact (recall $X$ is compact) and [1, page 465] (note $\overline{F(\overline{U \cap X})}$ is compact since $\alpha(F(\overline{U \cap X})$ ) $\leq$ $\alpha(\overline{U \cap X})=0)$ implies $F: \overline{U \cap X} \rightarrow C D(E)$ is u.s.c. Define the map $J$ by

$$
J(x)=\left\{\begin{array}{l}
\mu(x) F(x), \quad x \in \overline{U \cap X}, \\
\{0\}, \quad x \in X \backslash \overline{U \cap X} .
\end{array}\right.
$$

It is easy to see that $J: X \rightarrow C K(X)$ (see Theorem 2.1) is u.s.c. Then [4] implies that there exists $x \in X$ with $x \in J(x)$. As in Theorem 2.1 (a) we can easily deduce that $x \in F(x)$.

Remark 2.8. In Theorem 2.2, (2.8) could be replaced by (2.2) (note as well $U$ need not be convex) provided $X$ in (2.2) is such that $F(\overline{U \cap X})$ is relatively compact.

\section{EXISTENCE PRINCIPLES FOR OPERATOR EQUATIONS}

In this section let's begin by discussing the operator inclusion

$$
x(t) \in N x(t) \text { for } t \in[0, T] .
$$

Solutions to (3.1) will be sought in $C([0, T], E)$; here $E$ is a real Banach space.

Theorem 3.1. Let $E=(E,\|\cdot\|)$ be a Banach space and suppose there is a constant $M_{0}$, independent of $\lambda$, with $\|y\|_{0}=\sup _{[0, T]}\|y(t)\| \neq M_{0}$ for any solution $y \in C([0, T], E)$ to

$$
x(t) \in \lambda N x(t) \quad \text { for } t \in[0, T]
$$

for each $\lambda \in(0,1)$. Let

$$
U=\left\{u \in C([0, T], E):\|u\|_{0}<M_{0}\right\}
$$

and suppose

$$
N: \bar{U} \rightarrow C D(C([0, T], E)) \text { has closed graph }
$$

and

$$
\left\{\begin{array}{l}
N: \bar{U} \rightarrow C D(C([0, T], E)) \text { is a condensing map with } \\
N(\bar{U}) \text { a subset of a bounded set in } C([0, T], E)
\end{array}\right.
$$

are satisfied. Then (3.1) has a solution $y \in C([0, T], E)$ with $\|y\|_{0} \leq M_{0}$.

Remark 3.1. It is of interest to compare Theorem 3.1 with the results in [8] .

Proof. Apply Theorem 2.2 (notice $(A 2)$ with $x_{0}=0$ cannot occur) to deduce that (3.1) has a solution in $\bar{U}$.

A particular example of (3.1) will be the Volterra integral inclusion

$$
y(t) \in g(t)+\int_{0}^{t} k(t, s) F(s, y(s)) d s \text { for } t \in[0, T] .
$$

For notational purposes let $C(E)$ denote the family of nonempty, closed, convex subsets of $E$ and $C C(E)$ the family of nonempty, compact, convex subsets of $E$. 
Theorem 3.2. Let $E=(E,\|\cdot\|)$ be a separable Banach space with $F:[0, T] \times$ $E \rightarrow C C(E)$ and $k:[0, T] \times[0, t] \rightarrow \mathbf{R}$. Assume the following conditions are satisfied:

$$
\left\{\begin{array}{l}
\text { (i). } t \mapsto F(t, x) \text { is measurable for every } x \in E \\
\text { (ii). } x \mapsto F(t, x) \text { is u.s.c. for a.e. } \quad t \in[0, T]
\end{array}\right.
$$

$$
\left\{\text { for each } r>0 \text { there exists } h_{r} \in L^{1}[0, T] \text { such that }\|F(t, x)\| \leq h_{r}(t)\right.
$$

f for a.e. $t \in[0, T]$ and every $x \in E$ with $\|x\| \leq r$;

$$
\begin{aligned}
& \left\{\begin{array}{l}
\text { for each } t \in[0, T], k(t, s) \text { is measurable on }[0, t] \text { and } \\
k(t)=\text { ess sup }|k(t, s)|, 0 \leq s \leq t, \text { is bounded on }[0, T] ;
\end{array}\right. \\
& \left\{\begin{array}{l}
\text { the map } t \mapsto k_{t} \text { is continuous from }[0, T] \text { to } L^{\infty}[0, T] \\
\text { here } k_{t}(s)=k(t, s)
\end{array}\right.
\end{aligned}
$$

and

$$
g:[0, T] \rightarrow E \text { is single valued with } g \in C([0, T], E) .
$$

Let $K(t, s, u)=k(t, s) F(s, u)$ and assume

$$
\left\{\begin{array}{l}
\text { there exists a constant } \gamma \geq 0 \text { with } 2 \gamma T<1 \text { and with } \\
\alpha(K([0, T] \times[0, t] \times \Omega)) \leq \gamma \alpha(\Omega) \text { for any bounded } \\
\text { subset } \Omega \text { of } E
\end{array}\right.
$$

holds. Finally suppose there is a constant $M_{0}$, independent of $\lambda$, with $\|y\|_{0} \neq M_{0}$ for any solution $y \in C([0, T], E)$ to

$$
y(t) \in \lambda\left(g(t)+\int_{0}^{t} k(t, s) F(s, y(s)) d s\right) \quad \text { for } t \in[0, T]
$$

for each $\lambda \in(0,1)$. Thus (3.5) has a solution $y \in C([0, T], E)$ with $\|y\|_{0} \leq M_{0}$.

Proof. Let

$$
U=\left\{y \in C([0, T], E):\|y\|_{0}<M_{0}\right\} .
$$

In Theorem 2.1 of [9] we showed $N: \bar{U} \rightarrow C(C([0, T], E))$ has closed graph (see [9] for the definition of $N)$. Also using (3.11) we showed $N: \bar{U} \rightarrow C(C([0, T], E))$ is condensing. Apply Theorem 3.1 to deduce the result.

Remark 3.2. Notice (3.11) can be replaced by (see [9])

$$
\left\{\begin{array}{l}
\text { there exists a constant } \gamma \geq 0 \text { with } 2 \gamma T<1 \text { and with } \\
\alpha(K(\{t\} \times[0, t] \times \Omega)) \leq \gamma \alpha(\Omega) \text { for } t \in[0, T] \text { and any } \\
\text { bounded subset } \Omega \text { of } E .
\end{array}\right.
$$

Remark 3.3. If $k(t, s)=1,0 \leq s \leq t, 0 \leq t \leq T$ and $g(t)=x_{0}, t \in[0, T]$ (the differential inclusion case), then (3.11) can be replaced by (see [9])

$$
\left\{\begin{array}{l}
\text { there exists a constant } \gamma \geq 0 \text { with } 2 \gamma T<1 \text { and with } \\
\lim _{h \rightarrow 0^{+}} \alpha\left(F\left(J_{t, h} \times \Omega\right)\right) \leq \gamma \alpha(\Omega) \text { for } t \in[0, T] \text { and for } \\
\text { any bounded subset } \Omega \text { of } E \text {; here } J_{t, h}=[t-h, t] \cap[0, T] .
\end{array}\right.
$$


Remark 3.4. It is also possible to discuss the Hammerstein integral inclusion (see [9] ),

$$
y(t) \in g(t)+\int_{0}^{T} k(t, s) F(s, y(s)) d s \text { for } t \in[0, T]
$$

we leave the details to the reader.

\section{REFERENCES}

[1] C.D. Aliprantis and K.C. Border, Infinite Dimensional Analysis, Springer Verlag, Berlin, 1994. MR 96k:46001

[2] K. Deimling, Nonlinear Functional Analysis, Springer-Verlag, Berlin, 1985. MR 86j:47001

[3] K. Deimling, Multivalued Differential Equations, Walter de Gruyter, Berlin, 1992. MR 94b:34026

[4] P.M. Fitzpatrick and W.V. Petryshyn, Fixed point theorems for multivalued noncompact acyclic mappings, Pacific Jour. Math., 54(1974), 17-23. MR 53:8973

[5] M. Frigon, Théoremes d'existence de solutions d'inclusions différentielles, Topological Methods in Differential Equations and Inclusions (edited by A. Granas and M. Frigon), NATO ASI Series C, Vol 472, Kluwer Acad. Publ., Dordrecht, 1995, 51-87. MR 96m:34025

[6] H. Mönch, Boundary value problems for nonlinear ordinary differential equations of second order in Banach spaces, Nonlinear Anal., 4(1980), 985-999. MR 82c:34075

[7] D.O'Regan, A topological approach to integral inclusions, Proc. Royal Irish Acad., 97A(1997), 101-111.

[8] D.O'Regan, Abstract operator inclusions, Functional Differential Equations, 4(1-2)(1997), 143-154. CMP 98:07

[9] D.O'Regan, Multivalued integral equations in finite or infinite dimensions, Communications in Applied Analysis, 2(4)(1998), 487-496. CMP 98:16

[10] C.H. Su and V.M. Sehgal, Some fixed point theorems for condensing multifunctions in locally convex spaces, Proc. Amer. Math. Soc., 50(1975), 150-154. MR 52:1430

Department of Mathematics, National University of Ireland, Galway, Ireland

E-mail address: donal.oregan@nuigalway.ie 